\title{
How to Create Engaging Experiences From Human Emotions
}

\section{Maura Mengoni}

Università Politecnica delle Marche

m.mengoni@univpm.it

ORCID 0000-0003-2826-7455

\section{Luca Giraldi}

Università degli Studi di Macerata

l.giraldi@unimc.it

ORCID 0000-0002-3446-8993

\author{
Silvia Ceccacci \\ Università Politecnica delle Marche \\ s.ceccacci@pm.univpm.it \\ ORCID 0000-0001-8916-1262
}

\section{Roberto Montanari}

Università Degli Studi Suor Orsola Benincasa

roberto.montanari@unisob.na.it

ORCID 0000-0002-8242-0521

\begin{abstract}
There are several situations where the success of a product or a service depends on people's emotional reactions while interacting with it. Feelings depends on the personal reaction to the values it offers. Therefore, it is not possible to presume that all persons will share the same reactions. An important challenge that designers have to face is to measure such feelings at every digital and physical touchpoint encountered by the customer and in which an experience takes place.

In this context, the present research proposes an emotional-sensitive system able to measure the customer's emotion and behavior and responsively adapt the experience to generate an individualized human-system interaction. It enables a continuous flow between analysis and reaction at every touchpoint of the customer experience. The paper illustrates the implementing technological framework, developed by EMOJ, an Italian startup leader in Artificial Intelligence (Al), and several applicative use cases.
\end{abstract}

\author{
Keywords \\ Emotion \\ Face coding \\ Customer experience \\ Human-Centered Design \\ Human-Machine Interaction
}




\section{Introduction}

Today the quality of a product and the corresponding user experience it provides are necessary, even not sufficient, to ensure product success on the market (Van de Sand et al., 2019). To keep up their competitiveness, companies need to include their product and/or services as part of a well-conceived and comprehensive Customer eXperience (CX). Most western economies continue to shift from providing discrete products to providing and orchestrating a bunch of services around one or more products to realize the CX. Designers have therefore moved their input "upstream" to shape the companies' business models and design every touchpoint where people and company meet each other.

Once these "meetings" take place, customers have different levels of reaction, from the very first contact to the activation of a deeper and analytical approach started by their cognitive apparatus. Donald Norman (2004) identified three levels that users would activate once they meet a product and/or service. The first one is called visceral, and it states our first impression, where most of us decide if the attitude to this product or service is positive or critical. The others (behavioural and reflective) take place once the relationship is established, and they address the operational interaction with the product/service. Daniel Kahneman identified two parts of our brain (systems 1 and 2) that intervene in such experiences (Morewedge \& Kahneman, 2010). System 1 (fast) creates an immediate awareness in the customers, which quickly provides a set of decisions regarding the product and/or services, most emotional and intuitive. System 2 (slow) comes later and makes every decision more analytical, rational, data-driven, and sometimes inconsistent with the first (see the so-called "creative paradox", Rizzo, 2020).

It seems widely agreed among researchers that the first impression is quick, primarily impressive and emotional, and most of our other relation is based on this. Therefore, as this impression is so important, a detailed understanding of how it takes shape and works is crucial for the design of every CX clue. Behavioural-based research seems to show difficulties in understanding this type of reaction using traditional tools such as interviews and questionnaires (Lajante \& Ladhari, 2019). However, measurement is a relevant challenge as understanding people's specificity via emotional reactions could create a data ground to which a more aware and people-centric design could take shape to promote better product, service, and system.

Once the first contact has been established, the CX continues, and in every further step, there is a combination of cognitive assessments and emotional reactions (Verhulst et al., 2020). Some studies on usability and user-centred product design indicate a strong relationship between functionality, usability, and aesthetics. For instance, some interactive products, such as an ATM, were evaluated as more usable because of their look and feel. In automotive, negative emotions such as fear could critically impact on speed and vehicles' controllability (Braun et al., 2019). Therefore, a further challenge to face is understanding the role of emotions in guiding the interaction between users and product/ systems at every touchpoint. The objective measurement of the 
emotional state represents an essential topic in computer science. The following chapter discusses in detail the available techniques. It introduces the one proposed by the Italian startup EMOJ that has had the merit of integrating emotional coding into a continuous flow of human analysis and adaptation of the human-system interaction. After a brief overview of the proposed technological framework, a set of use cases to demonstrate the challenging approach in CX design is presented.

\section{Literature Overview on Emotion Measurement}

Among the current technologies that could take the challenge to promote measurements of feelings, emotions and behaviours for the designer, camera-based sensors, equipped with machine learning and artificial intelligent algorithms, seems to be the most promising ones (Karyotis et al., 2017). Today, cameras are everywhere, so that they have become an integral part of our daily lives. This makes the camera a sensor of choice for collecting consumer experience information in a non-invasive way. Thanks to the information that can be extrapolated from the videos through machine learning, it is now possible to have a huge amount of video-related information.

The literature proposes several methods based on image processing and Convolution Neural Networks (CNN) that allows determining user gender and age, such as proposed in Ceccacci et al. (2018). Some studies tested methods, based on regression and CNN, to track a person eye-gaze by using standard webcams or camera phones (Generosi et al., 2020; Krafka et al., 2016; Papoutsaki et al., 2016).

By analyzing face images from the video, it is possible to identify people's emotions. Many studies proposed methods to recognize patterns from facial expressions and to connect them to emotions. Most of them allow the recognition of Ekman \& Keltner's primary emotions (1970), such as fear, anger, joy, surprise, etc. They take in different input kinds of pictures and make predictions according to the trained model. Using multiple cameras can also track multiple persons' positions and actions within an environment (Arsic et al., 2008).

The above review of the literature highlights how the measurement of emotions and behaviours is possible in each touchpoint. A key point becomes to determine when and where to use this information and how to introduce it in designing and creating a unique, individualized customer experience.

\section{The New Design Approach Based on Complex Human State Monitoring}

\section{A Continuous Flow Between Analysis and Reaction}

The discussion on the current challenges in customer experience highlighted how strategic had become the understanding of the customers' emotions and their mapping with their behaviour during the interaction with every touchpoint along the customer journey. 
However, if this knowledge is not reported operationally in constructing an adaptive customer experience, it could be helpful only for investigative purposes and for orienting the creative design processes. Therefore, the goal is to exploit data about human monitoring to make services, human-machine interfaces, and all living environments adaptable to human emotions to realize a more satisfying, engaging and empathetic experience.

The introduction of emotions in a continuous flow of analysis-reaction-feedback requires, on the one hand, the availability of an interconnected network of sensors and actuators, i.e., Internet of Things (loT), distributed in the environment and embedded in products, and on the other hand, the development of an emotional-sensitive system to responsively adapt to the user to generate individualized experiences. In addition, upstream in the design process, the designer must consider the degree of product interactivity ensured by the current broad penetration of loT technologies and then provide a certain level of flexibility to make it responsive. While this last aspect relies on the designer's mindset change and expertise on technologies, the first one can be reached by proposing a new design approach enabling the management of customer experience strategies.

A customer-centred iterative perspective characterizes the approach and consists of the following steps:

1 Analysis of customers along their journey: in terms of customer's behaviours and emotional state during the interactions with any clue, characterizing the touchpoints (e.g., products, services, advertisements). This requires a real-time emotional recognition platform able to trace and analyze the customer along the multi-channel journey and to acquire valuable information for predicting the level of customer satisfaction (e.g., customer emotions, postures, gestures) in a non-intrusive way. The results are represented by constructing the customer journey map, which represents the store's main touchpoints, and the emotional curve to graphically show the level of customers' satisfaction and recognize which touchpoints need to be redesigned or adapted.

$2 \quad$ Planning of strategies to improve the customer experience: define all changes to any touchpoint to personalize the customer experience. Data collected during the analysis stage can be processed to map the customers' behavioural responses to the main features of the touchpoints. In this way, it is possible to determine the configuration corresponding to the higher level of customer satisfaction for each customer stereotype. This supports the definition of touchpoint adaptation requirements more objectively and rationally.

3 Development of all individualized experiences for every critical touchpoint: this may require redesigning products and services to realize interactive systems and adapt their features based on the user interaction experience. To this end, proper adaptation rules can be automatically generated and managed through machine learning algorithms through the data knowledge base previously acquired. 
Implementation, testing and evaluation: along the customer journey and definition of possible improvements (e.g. varying the order of the stimuli, proposing design guidelines to make the product more pleasant or the journey more attractive). Results include the elaboration of data collected through the construction of the emotional curve and guidelines to improve the $\mathrm{CX}$.

\section{EMOJ Framework}

Implementing the proposed holistic approach contributes to advancing state of art both in design and computer science. It requires an interconnected, integral and flexible technological framework enabling all conceptual steps, from analysis to adaptive reactions to user feedback, to effectively transform the recognized customers' feelings from negative to positive, intercept their wishes and respond in real-time to make them more satisfied.

In 2017, EMOJ was born as a spin-off of the Polytechnic University of Marche to provide designers and digital strategists with a technological framework to realize such an approach. The framework is based on three modules, one to analyze all human-system interactions at every touchpoint, one to know some essential metrics to assess the CX Fig. 1, one to decide and then react in real-time to change the human state to realize a pleasant, engaging, and personalized experience Fig. 2.

The first module allows the acquisition of several types of information related to the customers' behaviour. It implements face coding, facial expressions recognition, eye-gaze tracking, head tracking, body posture tracking Fig. 3. The second provides a collection of intelligent analytics tools that quickly and efficiently enable data processing to extrapolate valuable insight to understand better the touchpoint features and clues that most affect the customer experience. The last exploits the system described in Ceccacci et al. (2018). It implements Al algorithms based on inductive inference, making decisions based on logical rules derived from a knowledge base defined through the relations between customers profiles and the adaptable feature of products/services/clues.

\section{Case History}

The multidisciplinary nature of the approach and the cross-applicability of the technology can be demonstrated by numerous applications of the EMOJ framework, illustrated as follows.

\section{Audience Measurement}

The first application is the system used for audience measurement in opera live shows, in the open-air neoclassical theatre Arena Sferisterio, in Macerata.

Infrared cameras were positioned in the Sferisterio arena to detect the facial audience expression during the show. The soft- 

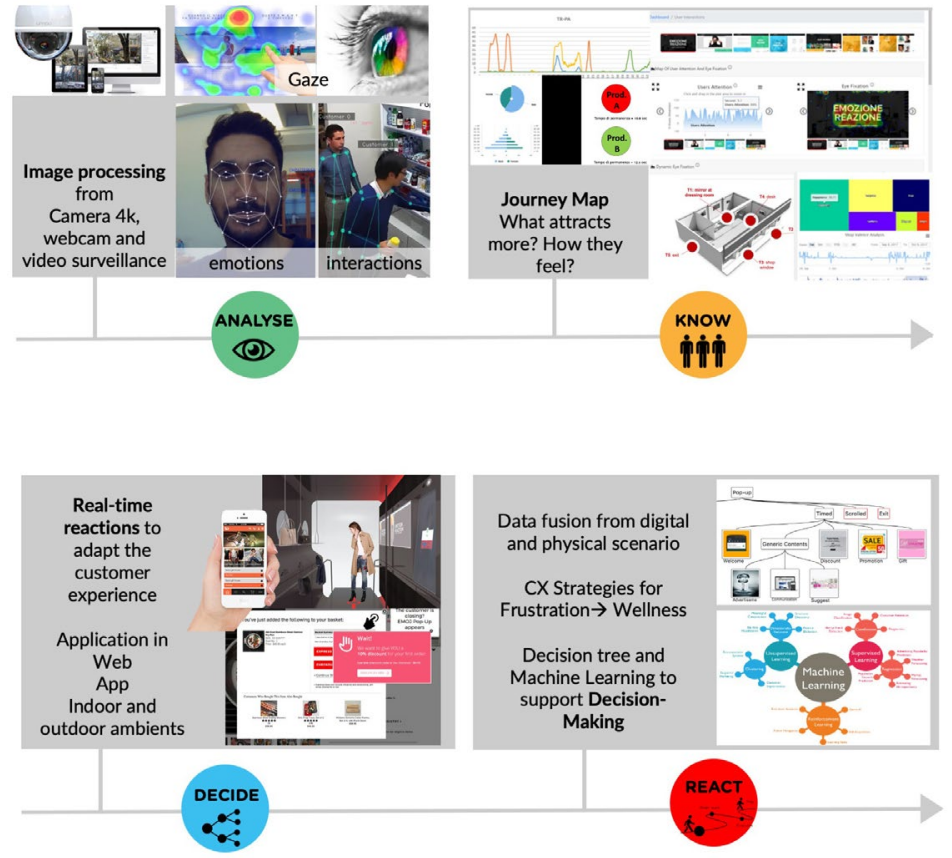

ware adopted in this context implements a combination of facial recognition and gaze tracking technologies based on artificial intelligence algorithms, as described in detail in Generosi et al. (2020) and Talipu et al. (2019). It enables the age and gender recognition of people shot by the cameras, monitoring their emotional state and corresponding level of interest and involvement. Collected data were elaborated to analyze the dynamics of the audience's emotional behavior during the representations to investigate spectators'level of customer satisfaction. Results evidenced that collected data related to audiences can be helpful for the artistic director to estimate the audience's overall level of satisfaction and better guide the choices regarding the specific characteristics of the performance and casting, to improve the artistic offering.

\section{Remote Usability Testing}

The technology can also be embedded in mobile apps and websites to monitor the user experience and automatically find usability issues. The developed remote usability testing platform tracks user interactions such as scrolls and clicks, plots gaze and find heatmaps, assesses the satisfaction rate, and completely maps the user journey. It allows designers to quickly, automatically and in remote modality assess user behaviour to improve app usability. The platform has a dashboard to visualize usability tests' results and manage participants. Standard analytics only answers "what", while our UX track can answer "why".
Fig. 1

The technological framework: the two modules for analyzing and understanding the complex human state.

Fig. 2

The technological framework: the module to create adaptive reactions to human state. 


\section{:E MO J:}
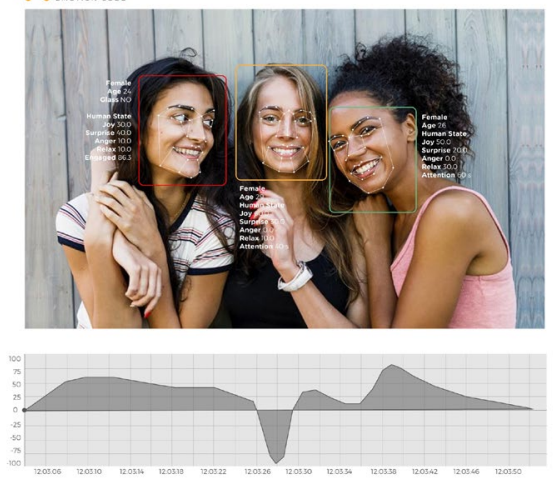

Number 3

Average Age 27 years old

Gender 100 Female O Male

Attention $30 \mathrm{sec}$

$\begin{array}{ll}\text { Joy } 11.6 & \text { Disgust } 0.0 \\ \text { Surprise } 83.4 & \text { Anger } 0.0 \\ \text { Neutral } 5.0 & \text { Fear } 0.0 \\ \text { Satisfaction } 80 \% & \text { Engagement 35\% }\end{array}$

Emotional Trend

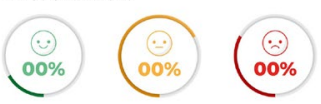

A Multisensory Touchpoint in Retail:

The Evoque Tunnel

The application showed in Figure 4, is an emotional tunnel sensed by multiple cameras and equipped with LED lighting, LCD displays, video projectors and audio speakers. When a person enters the tunnel, she/he is recognized, and a customized experience is offered. Lights change according to her/his emotions; a video mapping appears, and every stimulus changes in real-time. The Evoque tunnel was built and installed at the Retail Forum in Milan, 2018 to communicate a famous luxury shoe brand.

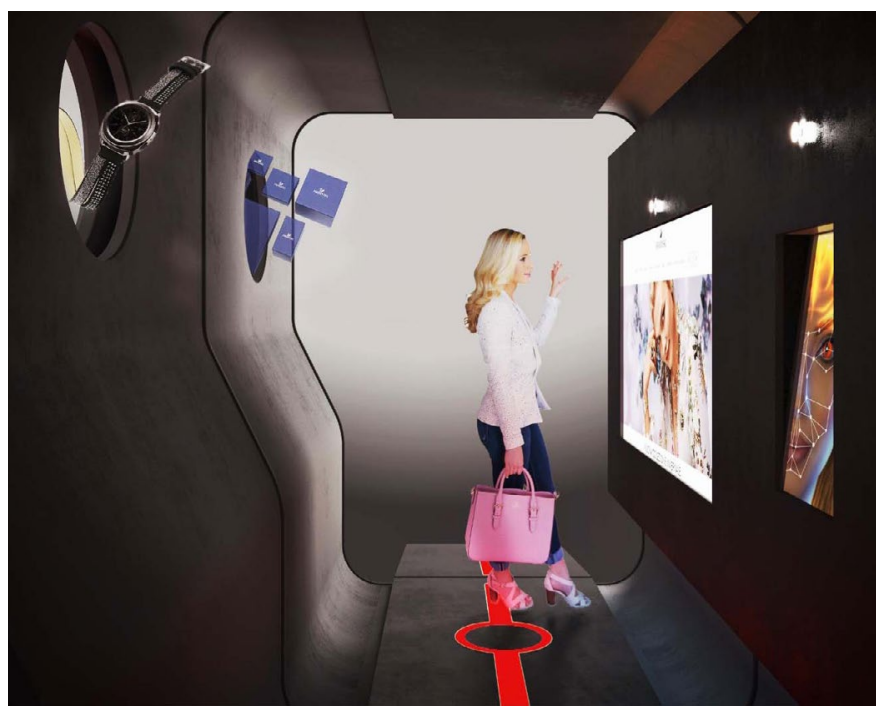

Fig. 4

The Evoque tunnel concept.

Adaptive Multimedia Totem for Museums

The second application is a multimedia totem for museums equipped with a webcam on the top. When visitors are in front of it, EMOJ captures their age, gender and emotions and is associated with a specific museum cluster, such as annoyed visitors, curious visitors or thought- 
ful people. Based on the cluster, the system proposes a journey across the museum by visualizing different artworks. At the end of the digital experience, visitors can download a map of the proposed journey and start with the visit. The totem was placed at the entrance of the rooms that host the exhibition of Modern Art of Palazzo Buonaccorsi. The application was available to visitors for two months, during which the experimentation was carried out. A total of 1976 experiences related to one or more people has been analyzed. Results reported in Altieri et al. (2021) evidence how the totem worked to create an interactive and emotional link with the groups, positively influencing their mood in the Pre-Experience phase and the subsequent Post-Experience phase. In particular, they highlight that the proposed system, aimed at acting as emotional leverage, has improved the positiveness of the emotions experienced by the visitors.

\section{Adaptive HMI for Automotive: Hu-Drive Technology}

Many of today's vehicles are equipped with Advanced Driver Assistance Systems (ADAS) that automatically recognize and react to potentially risky events in the driving scenario. A further step towards safety could be taken by addressing what is most human: driver's emotions. Intense, overwhelming emotions can impair the driver's judgement and effectiveness. This preamble has guided the development of a concept that EMOJ, in cooperation with RE: Lab (an Italian company focused on Human Machine Interface design), created and presented at CES 2021. The Hu-Drive (the name) is a human driver assistant system that dynamically adapts Human-Machine Interface to the driver's emotional state. The HU-Drive solution consists of two modules: module 1 recognizes the driver's emotional and cognitive state from images which are captured by cameras inside the cockpit and processed by Deep Neural Networks; module 2 is a set of HMI adaptation strategies that are triggered once a specific state is detected, to help the driver manage emotions and act safely. Anytime a critical state is detected, HMI can nudge the driver into controlling her emotions and thereby acting safely. Preliminary tests of the HU-Drive technology have been published in Ceccacci et al. (2020).

Fig. 5

The HU-Drive architecture.

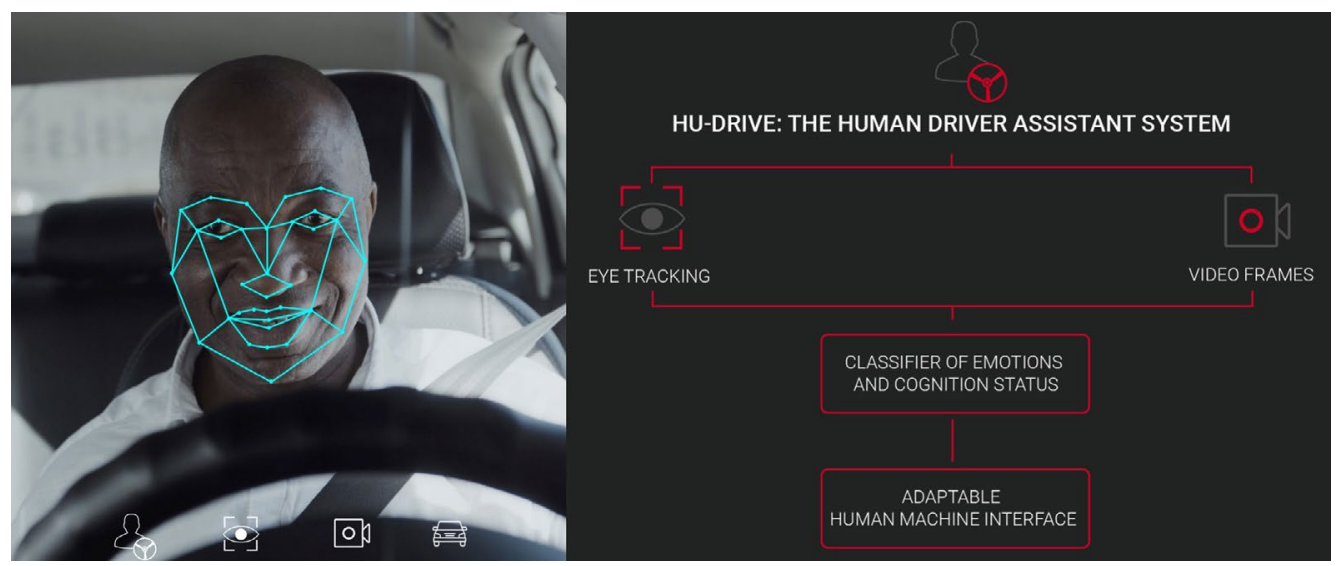




\section{Advancement in Distance Learning} and Digital Events

EMOJ takes the opportunity of Covid-19 to study and launch a product line dedicated to distance learning and, after its success, streaming events. Corsincloud is an e-learning platform to deliver compulsory professional training courses. It implements the system described in Ceccacci et al. (2021), which exploits the most recent developments in Deep Learning and Computer Vision for Affective Computing in compliance with the European GDPR. When the user starts to watch a video lesson, a tool is launched, accessing the frontal camera. Corsincloud performs continuous student's authentication based on face recognition, monitors the student's level of attention through head orientation tracking and gaze detection analysis, estimates student's emotions during the course attendance, and checks the number of people captured by the camera. With similar functionalities, two other solutions have been developed: Esamincloud and Eventincloud. Esamincloud also includes functionalities of a lockdown browser to check that students are not copying during the online exam. Eventincloud is a live event monitoring software (meetings, conferences, seminars) that measures the host user's engagement rate, satisfaction, and interaction level in real-time.

\section{Conclusion}

Today's scenario has rapidly changed to adapt products, services and experiences to the customers. However, the role of CX is not only determined by a behavioural harmonization between users' initiatives, actions and reactions, and every touchpoint they meet while accessing a product, service or a combination of both. On the contrary, other elements seem to be crucial in activating and keeping such relations, among which emotions play a strategic role.

Emotions are crucial as they influence behaviour and cognition in deciding to act or not, perceiving and interpreting something, and orienting and prioritizing our attention. Therefore, the emotional understanding is not only a new research perspective since the publication of the Rosalind Picard book on affective computing (Picard, 1997), but is going to be a driver for the design and development of products, services and in general, whatever is depicting a CX. Emotions are driving the first connection with products and services, orienting the decision we take in assessing if something is good or bad, and based on this, emotions will guide the next steps in the experiences with products and services.

Therefore, the support of the emotions in the design is crucial. However, the technologies should detect real-time users' emotional status and govern the adaptation of their mutual interaction. Moreover, this is precisely the approach followed by EMOJ. This technological framework detects emotional status. This intervenes in adapting the quality and nature of services in a public event as an Opera, in a museum interaction experience, in the vehicle $\mathrm{HMI}$ or the support for distance learning and assessment.

The first results on the use cases presented have provided a solid set of empirical assessments of this technological framework.
Maura Mengoni

Associate Professor in Industrial Engineering Design and Methods Università Politecnica delle Marche. Responsible for the Virtual Reality Lab. She authored more than 120 international publications. Her primary research topics are Virtual Prototyping, Reverse Engineering, Collaborative Environments, User-Centred Design, Customer Experience (CX).

Silvia Ceccacci Ph.D. Fellows - Università Politecnica delle Marche. She is the author of more than 40 international publications. Her research topics include Human Factors \& Ergonomics, virtual prototyping, Experience Design, emotion aware and adaptive systems, and integrated approaches to exploit the value of Big data for servitization.

\section{Luca Giraldi}

Ph.D. and co-founder of EMOJ, Innovation Manager of MISE, received the MS Degree in Political Science in 2003, the BS Degree in Communication Science in 2005 at the Università di Macerata in 2005. He is an expert in customer experience, artificial intelligence and face coding. $\mathrm{He}$ is the author of more than twenty international publications and three patents.

\section{Roberto Montanari}

Ph.D., is co-founder and $R \& D$ responsible for RE:Lab, a company focused on Human Machine Interface design and development. He is a Professor of Interaction Design at Università Degli Studi Suor Orsola Benincasa (Naples) and technical director of the university research centre Scienza Nuova, focused on integrating humanities and technologies. 
What emerged in experimentations is that realizing the technological framework in real scenarios still requires substantial effort for designers and software developers, respectively, to design customized nudges and emotion regulation strategies suited for the specific use case and the implementation of decision-making rules. Future work will be focused on creating a more general set of nudges and related rules to make automatic and smart their selection by the system. However, the presented approach constitutes a new tool for designers and for a design process that - being aware of emotional reaction - could guide the creation of products and services even more customer-centric, and in the end, more empathetic.

\section{References}

Altieri, A., Ceccacci, S., Giraldi, L., Leopardi, A., Mengoni, M., \& Talipu, A. (2021). Affective Guide for Museum: A System to Suggest Museum Paths Based on Visitors' Emotions. International Conference on Human-Computer Interaction (pp. 521-532). Springer.

Arsic, D., Hristov, E., Lehment, N., Hornler, B., Schuller, B., \& Rigoll, G. (2008). Applying multi-layer homography for multi-camera person tracking. ACM/ IEEE International Conference on Distributed Smart Cameras (pp. 1-9). IEEE.

Braun, M., Schubert, J., Pfleging, B., \& Alt, F. (2019). Improving driver emotions with affective strategies. Multimodal Technologies and Interaction, 3(1), 21. 10.3390/mti3010021

Ceccacci, S., Generosi, A., Cimini, G., Faggiano, S., Giraldi, L., \& Mengoni, M. (2021). Facial coding as a means to enable continuous monitoring of student's behavior in e-Learning. Proceedings of the First Workshop on Technology Enhanced Learning Environments for Blended Education (teleXbe2021).
Ceccacci, S., Generosi, A., Giraldi, L., \& Mengoni, M. (2018). Tool to Make Shopping Experience Responsive to Customer Emotions. International Journal of Automation Technology, 12(3), 319-326.

Ceccacci, S., Generosi, A., Giraldi, L., Carbonara, G., Castellano, A., Montanari, R., \& Mengoni, M. (2020). A Preliminary Investigation Towards the Application of Facial Expression Analysis to Enable an Emotion-Aware Car Interface. In M. Antona, \& C. Stephanidis (Eds.), Universal Access in $\mathrm{Hu}$ man-Computer Interaction. Applications and Practice. Lecture Notes in Computer Science (pp. 504-517). Springer. 10.1007/978-3030-49108-6_36

Ekman, P., \& Keltner, D. (1970). Universal facial expressions of emotion. California Mental Health Research Digest, 8(4), 151-158.

Generosi, A., Ceccacci, S., Faggiano, S., Giraldi, L., \& Mengoni, M. (2020). A toolkit for the automatic analysis of human behavior in $\mathrm{HCl}$ applications in the wild. Advances in Science, Technology and Engineering Systems, 5(6), 185-192. 10.25046/aj050622.
Karyotis, C., Doctor, F., labal, R., \& James, A. (2017). Affect aware ambient intelligence: current and future directions. In A. Azira et al. (Eds.), State of the Art in Al Applied to Ambient Intelligence (pp. 8-67). IOS Press.

Krafka, K., Khosla, A., Kellnhofer, P., Kannan, H., Bhandarkar, S., Matusik, W., \& Torralba, A. (2016). Eye-tracking for everyone, in Proc. of the IEEE Computer Society Conf. on Computer Vision and Pattern Recognition, 2176-2184.

Lajante, M., \& Ladhari, R. (2019). The promise and perils of the peripheral psychophysiology of emotion in retailing and consumer services. Journal of Retailing and Consumer Services, 50, 305-313.

Morewedge, C.K., \& Kahneman, D. (2010). Associative processes in intuitive judgement. Trends in cognitive sciences, 14(10), 435-440.

Norman, D.A. (2004). Emotional Design. Basic Books.

Papoutsaki, A., Sangkloy, P., Laskey, J., Daskalova, N., Huang, J., \& Hays, J., (2016). Webgazer: Scalable webcam eye-tracking using user interactions. Proceedings of the Twenty-Fifth International Joint Conference on Artificial Intelligence-IJCAI.
Picard, R. (1997). Affective Computing. MIT Press.

Rizzo, A. (2020). Ergonomia Cognitiva. II Mulino.

Talipu, A., Generosi, A., Mengoni, M., \& Giraldi, L. (2019). Evaluation of Deep Convolutional Neural Network architectures for Emotion Recognition in the Wild. 2019 IEEE 23rd International Symposium on Consumer Technologies, ISCT (pp. 25-27). 10.1109/ ISCE.2019.8900994

Van De Sand, F., Frison, A.K., Zotz, P., \& Holl, K. (2019). The Intersection of User Experience (UX), Customer Experience (CX) and Brand Experience (BX). User Experience is Brand Experience, Management for Professionals book series (pp. 71-93). Springer.

Verhulst, N., Vermeir, I., Slabbinck, H., Lariviere, B., Mauri, M., \& Russo, V. (2020). A neurophysiological exploration of the dynamic nature of emotions during the customer experience. Journal of Retailing and Consumer Services, 57. https://doi.org/10.1016/j. jretconser.2020.102217 